\section{NOVEL DNAM-1 AXIS MEMBER, PVRIG, IS POTENTIALLY A DOMINANT CHECKPOINT INVOLVED IN STEM-LIKE MEMORY T CELLS - DENDRITIC CELL INTERACTION}

Zoya Alteber, Gady Cojocaru, Masha Frenkel, Emmanuel Weyl, Niv Sabath, Assaf Wool, Amit Novik, Yossef Kliger, Zurit Levine, Eran Ophir*. Compugen Ltd., Holon, Israel

Background T-cell accumulation in tumors is a prerequisite for response to cancer immunotherapy. Recent studies highlighted the importance of an early-memory (stem-like) T-cell sub-population, that can self-renew and differentiate into effector cells, and of dendritic cells (DCs), which are essential for T-cell priming and expansion following checkpoint blockade. ${ }^{1}{ }^{2}$ PVRIG is a novel inhibitory receptor that competes with the co-activating receptor DNAM-1, for the binding of a shared ligand, PVRL2. PVRIG expression is induced on $\mathrm{T}$ and NK tumor infiltrating cells, whereas PVRL2 is expressed on tumor, endothelial and myeloid cells in the tumor micro-environment (TME). ${ }^{3}$ We investigated the expression of PVRIG and PVRL2 across TME immune subpopulations.

Methods Publicly available TME scRNA sequencing datasets were analyzed for the expression of PVRIG and PVRL2 across immune subsets. Unsupervised principal-component-analysis and hierarchical co-expression pattern among genes known to be expressed on naïve, memory, and exhausted CD8 + T-cells was performed. Observations were validated by flow-cytometry and immunohistochemistry analysis across a variety of tumor indications. Proximity Extension Assay (PEA, Olink) was conducted using serums collected at several time-points from COM701 (anti-PVRIG antibody) and nivolumab treated patients in a Phase-1 study (NCT03667716).

Results Across scRNA datasets, PVRIG, like TIGIT and PD-1, was expressed by both stem-like (TCF1+PD1+) and exhausted (TIM3+CD39+) CD8 + T-cells. High resolution unsupervised scRNA gene co-expression analysis revealed that while TIGIT is strongly correlated with PD-1, CTLA-4, and other markers of exhausted T-cells, PVRIG uniquely clusters with markers of early memory T-cells (figure 1). Accordingly, PVRIG protein expression was increased on CD28+ earlymemory T-cells across indications (figure 2).RNA expression data also revealed that PVRL2 is more abundantly expressed across DC-subtypes compared to PD-L1 and PVR (ligand of TIGIT, figure 3). Flow cytometry confirmed dominant PVRL2 expression on DC subtypes across tumor indications. Immunohistochemistry analysis identified PVRL2 expression in Tertiary Lymphoid Structures (figure 4). Finally, preliminary analysis of serum from COM701+nivolumab treated patients revealed elevated induction of activated-DC markers in two patients that responded clinically (RECIST criteria), compared to nonresponders (figure 5).

Conclusions PVRIG is co-expressed with PD-1 and TIGIT on stem-like and exhausted $\mathrm{T}$ cells but has a unique dominant expression on early memory cells, while PVRL2 is abundantly expressed across DC-types. PVRIG blockade could therefore enhance memory T-cells activation by DCs, resulting in their increased expansion and differentiation. Accordingly, early data shows increased induction of activated DC markers, potentially following efficient T-DC interaction, in serum of two patients responding to COM701+nivolumab.
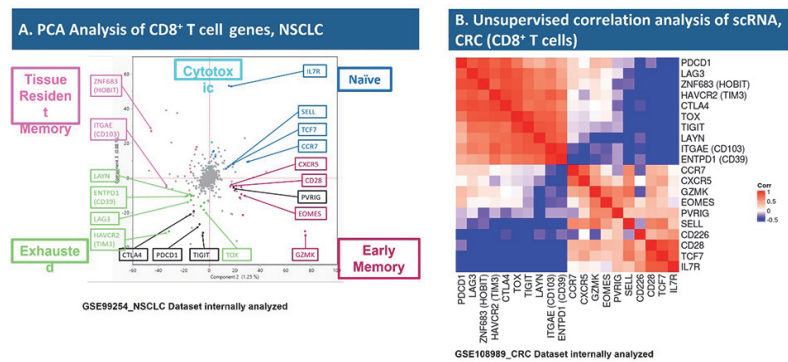

Abstract 252 Figure 1 PVRIG clusters with early differentiation/ memory genes, unlike other immune checkpoints that cluster with exhausted genes, in CD8+ T cells. (A) Unsupervised PCA analysis was performed on a scRNA expression matrix of TME CD8+ T cells, which includes all variable genes. Using cells as features and genes as entries, hierarchical co-expression pattern among genes known to be expressed on naïve, memory, and exhausted CD8+ T-cells was performed. (B) scRNA-Seq datasets were analyzed for co-expression pattern among 19 genes, including genes known to be expressed on naïve (TCF7, IL7R, SELL), memory (GZMK, EOMES), and exhausted (PDCD1, LAG3, HAVCR2) CD8 T cells. Average gene-gene correlation over all datasets was calculated. Representative dataset of $n=13$ (CRC, NSCLC, HNSCC, Melanoma, Liver cancer) is presented.
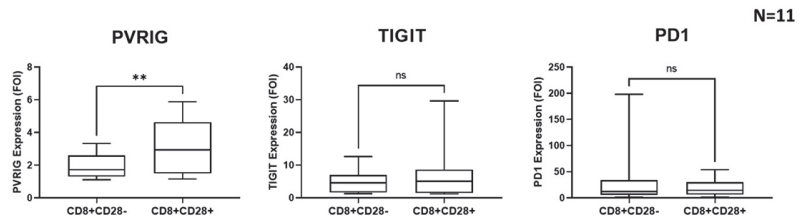

Abstract 252 Figure 2 PVRIG is expressed by early memory CD8+ T cells in the TME. Samples $(n=11)$ of CRC, ovarian and bladder cancer were dissociated to single cell suspensions and analyzed for gene expression by flow-cytometry. Paired T-test was used to compare between PVRIG expression among cell populations

A.

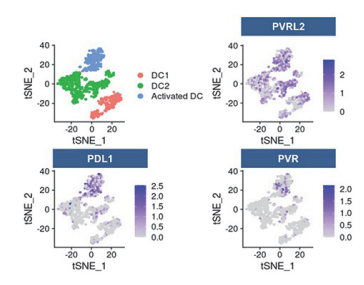

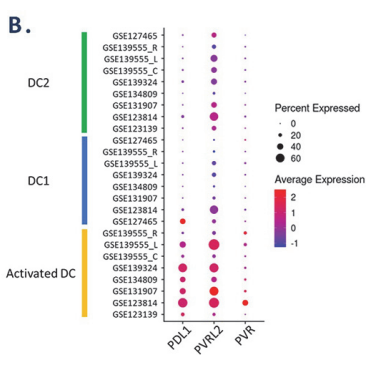

B.

Abstract 252 Figure 3 PVRL2 is dominantly expressed on dendritic cells. (A) tSNE map depicting the expression profile of PVR/PVRL2/PDL1 in major dendritic cell subsets in Basal Cell Carcinoma patients. (B) Dot plots showing the percent of cells and average level of expression of PVR/PVRL2/PDL1 in major dendritic cell subsets across multiple scRNA cancer studies. 


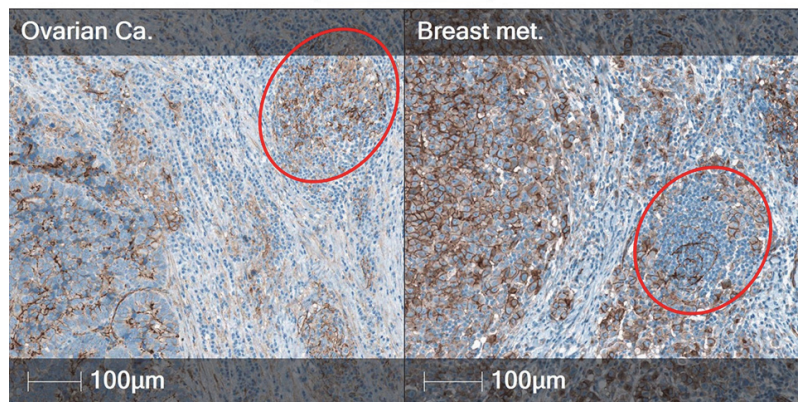

Abstract 252 Figure 4 PVRL2 is expressed in tertiary lymphoid structures in the tumor bed. Tertiary Lymphoid Structures (TLSs) were identified in subsets of samples across all tumors tested (NSCLC, CRC primary and metastasis, ovarian cancer, endometrial cancer, breast primary TNBC and breast metastasis) and for most cases TLSs were positive for PVRL2. Staining was preformed using a proprietary rabbit $\mathrm{mAb}$ raised against the ECD of PVRL2 on a Dako Autostainer
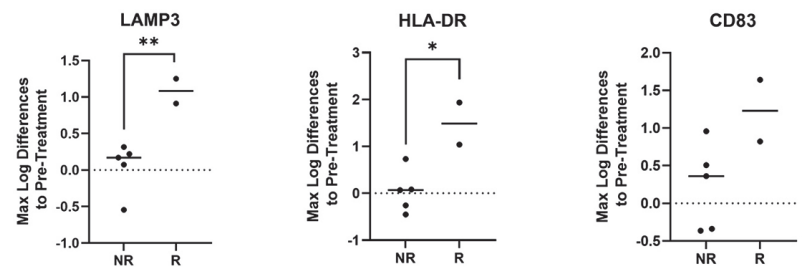

Abstract 252 Figure 5 Elevated induction of activated-DCs markers in patients that clinically responded to COM701+nivolumab, compared to non-responders. Serum of 7 patients from the nivlumab+COM701 dose escalation arm, were analyzed using Olink Explore 1536. For each patient, the difference between all on-treatment time points to pretreatment were calculated. Group difference based on response, RECIST criteria (responders $(R)$ : $C R+P R$ vs. non responders (NR): $S D+P D$ ) were compared by student t-test for all available time points grouped

\section{REFERENCES}

1.. Jansen CS, Prokhnevska N, Master VA, et al. An intra-tumoral niche maintains and differentiates stem-like CD8 T cells. Nature 2019;576:465-470.

2.. Held $W$, et al. Intratumoral CD8+ T cells with stem cell-like properties: implications for cancer immunotherapy. Sci Trans/ Med 2019;11(515):eaay6863

3.. Whelan S, Ophir E, Kotturi MF, Levy O, Ganguly S, Leung L, et al. PVRIG and PVRL2 are induced in cancer and inhibit CD8+ T-cell function. Cancer Immunol Res 2019;7:257-68.

Ethics Approval

Clinical trial identification NCT03667716The study was approved by each site's ethics board.

\section{Consent}

Clinical trial identification NCT03667716Written informed consent was obtained from the patient for publication of this abstract and any accompanying images. A copy of the written consent is available for review by the Editor of this journal.

http://dx.doi.org/10.1136/jitc-2021-SITC2021.252 